\title{
Using linked administrative and disease- specific databases to study end-of-life care on a population level
}

\author{
Arno Maetens ${ }^{1 *+} \mathbb{D}$, Robrecht De Schreye ${ }^{1 \dagger}$, Kristof Faes $^{1,2+}$, Dirk Houttekier ${ }^{1}$, Luc Deliens ${ }^{1,3}$, Birgit Gielen ${ }^{4}$, \\ Cindy De Gendt ${ }^{5}$, Patrick Lusyne ${ }^{6}$, Lieven Annemans ${ }^{2}$ and Joachim Cohen ${ }^{1}$
}

\begin{abstract}
Background: The use of full-population databases is under-explored to study the use, quality and costs of end-of-life care. Using the case of Belgium, we explored: (1) which full-population databases provide valid information about end-of-life care, (2) what procedures are there to use these databases, and (3) what is needed to integrate separate databases.

Methods: Technical and privacy-related aspects of linking and accessing Belgian administrative databases and disease registries were assessed in cooperation with the database administrators and privacy commission bodies. For all relevant databases, we followed procedures in cooperation with database administrators to link the databases and to access the data.

Results: We identified several databases as fitting for end-of-life care research in Belgium: the InterMutualistic Agency's national registry of health care claims data, the Belgian Cancer Registry including data on incidence of cancer, and databases administrated by Statistics Belgium including data from the death certificate database, the socio-economic survey and fiscal data. To obtain access to the data, approval was required from all database administrators, supervisory bodies and two separate national privacy bodies. Two Trusted Third Parties linked the databases via a deterministic matching procedure using multiple encrypted social security numbers.

Conclusion: In this article we describe how various routinely collected population-level databases and disease registries can be accessed and linked to study patterns in the use, quality and costs of end-of-life care in the full population and in specific diagnostic groups.
\end{abstract}

Keywords: End-of-life, Data linkage, Administrative databases, Disease-specific databases, Full-population

\section{Background}

It has been argued that there is a particular challenge for end-of-life care research to develop a public health approach [1] which would include, among other things, the need for a focus on total populations instead of individuals at risk or those receiving a certain health care service. This means that many, often 'hidden', publics also need to be studied [1,2]. End-of-life care research indeed often suffers from selection bias, recall bias and

\footnotetext{
* Correspondence: Arno.maetens@vub.ac.be

${ }^{\dagger}$ Equal contributors

${ }^{1}$ End of Life Care Research Group, Vrije Universiteit Brussel (VUB), Brussels,

Belgium \& Ghent University, Ghent, Belgium

Full list of author information is available at the end of the article
}

non-response bias [3-5] and difficult-to-reach populations tend to be under-represented due to ethical and practical considerations [6].

Administrative data can provide a major opportunity in this respect. They allow not only the monitoring of usage, quality and costs of end-of-life care on a population level [7], but also identifying populations dying of or dying with a specific disease such as cancer, chronic obstructive pulmonary disease (COPD) or Alzheimer's disease in order to evaluate patterns of end-of-life care within and across different trajectories of dying [8]. Many healthcare institutions generate, store and exchange large amounts of individual patient data [9]. Increasing digitalization in recent years has further 
facilitated and improved this process [10]. Although big data serve administrative purposes particularly (e.g. billing, tracking of health care reimbursement) they can provide useful research material from a public health perspective [11]. They often have a well-defined population and include subgroups or difficult-to-reach populations [6]. Because administrative data registrations are usually standardized and continuously collected they enable trend analyses and longitudinal studies. Moreover, since the data have already been collected, they are relatively inexpensive when compared with original data collections $[2,12]$. The expanding availability and quality of data input make them increasingly interesting to use in health research. Although full-population databases have been used to study end-of-life care since the late nineties (e.g. in Australia [13] and Canada [14]), the use of such data in end-of-life care research is still under-explored.

End-of-life care researchers may face several challenges when using administrative data. Administrative data are, for instance, not specifically designed for research purposes and therefore not directly usable for the evaluation of quality of care or quality of dying. They are not structured in readily available variables for analysis and may often lack the essential diseasespecific or relevant socio-demographic information needed in end-of-life care research. Additionally, healthcare data, socio-demographic data, socio-economic data and clinical data gathered on every citizen are stored in separate databases that are owned and handled by different organizations. Also, data security and confidentiality must be publicly guaranteed when using administrative databases for healthcare research. The challenge is thus to collect, link, integrate, store and process them so that they provide a useful input for end-of-life care research.

Using the case of Belgium, we describe how several full-population data sources can be accessed, linked, handled and stored in order to obtain a rich database for evaluating the use, quality and costs of end-of-life care. Our research questions are: (1) what data and databases are available that provide information about end-of-life care, (2) what are the procedures to obtain/use these data, (3) what is needed to integrate separate databases, and (4) what variables are available in these databases to study use, quality and costs of end-of-life care.

\section{Methods}

To address our research aims we systematically collected the necessary information in four phases:

1. First, we had to identify what databases provide information on the health care use, quality and costs near the end of life. We aimed to retrieve healthcare use data from all decedents for the two years prior to their death. A group of end-of-life researchers and health economics experts explored what data are available on healthcare and medication use that additionally (1) allow identification of people dying with or from cancer, Alzheimer's disease or COPD; and (2) provide relevant socio-economic and demographic information that is known from literature to influence end-of-life care patterns. Health claims data were used as the starting point as they provide critical data about patterns in formal care and medication prescription at the end of life. Other administrative databases and disease registries were explored to supplement the health claims database.

2. Once the databases and the data handling organizations were identified, the associated access procedures and permissions as well as linking possibilities were explored.

3. To complete the linking procedure, technical aspects and privacy protection measures were determined, explored and followed.

4. Finally, we composed an overview of available variables through this process. We examined how they can be used to study use, quality and costs of end-of-life care.

\section{Results}

\section{Identification and selection of databases}

A total of seven population-level databases handled by three different organizations were identified as providing the necessary information (Table 1).

The Inter Mutualistic Agency (IMA) manages the databases that included all reimbursement data of health care consumption from all seven healthcare insurers. Since health insurance with one of these insurers is legally mandatory in Belgium, reimbursement data of all legal residents are available in the IMA database. Moreover, thorough quality procedures result in reliable usability of the database for healthcare research. The IMA manages three databases: (1) a population database containing socio-demographic data of all insured persons; (2) a health care database containing health care use and costs data of both ambulatory and hospital care and (3) a pharmaceutical database containing medication prescription and costs data. The databases thus provide information on an individual level across the entire Belgian population. The IMA databases contain no information regarding medical diagnoses or any disease specific information.

The Belgian Cancer Registry was identified as a database to identify people who died with cancer. All Belgian oncological care programmes of hospitals and laboratories for anatomic pathology are legally bound to register each new cancer diagnosis with the cancer registry. The latter manages a database with diagnostic information on all incidences of cancer i.e. date of diagnosis, type of 
Table 1 Overview of population-level databases identified as relevant for end-of-life care research

\begin{tabular}{|c|c|c|c|}
\hline Database administrators & Database name & Population & Information provided in database \\
\hline \multirow[t]{3}{*}{ Inter Mutualistic Agency (IMA) } & Population Database & \multirow[t]{3}{*}{$\begin{array}{l}\text { Every Belgian citizen who is a member of } \\
\text { one of the seven (compulsory) Belgian } \\
\text { sickness funds, information in Population } \\
\text { Database is updated twice each year from } \\
2002 \text { onwards }\end{array}$} & $\begin{array}{l}\text { Socio-demographic characteristics } \\
\text { (age, sex, date of death, place of } \\
\text { residence, family composition, use } \\
\text { of supportive measures) }\end{array}$ \\
\hline & Pharmanet Database & & $\begin{array}{l}\text { Medication supply characteristics } \\
\text { (substance, quantity, prescriber, } \\
\text { expenses, refunds, delivery date) }\end{array}$ \\
\hline & Medical Claims Database & & $\begin{array}{l}\text { Health and medical care use } \\
\text { characteristics (quantity of use, } \\
\text { reimbursement, supplier, supplier } \\
\text { institution, length of treatment) }\end{array}$ \\
\hline Belgian Cancer Registry & Cancer registry & $\begin{array}{l}\text { Every new cancer diagnosis of Belgian } \\
\text { residents, registered by oncological care } \\
\text { programs and laboratories for anatomic } \\
\text { pathology }\end{array}$ & $\begin{array}{l}\text { Diagnostic characteristics (date } \\
\text { of diagnosis, type of cancer, TNM } \\
\text { gradation) }\end{array}$ \\
\hline \multirow[t]{3}{*}{ Statistics Belgium } & Death certificate database & $\begin{array}{l}\text { Every Belgian decedent with a registered } \\
\text { death certificate }\end{array}$ & $\begin{array}{l}\text { Direct and indirect causes of } \\
\text { death (in ICD-10 codes), socio- } \\
\text { demographics about the deceased, } \\
\text { place of death }\end{array}$ \\
\hline & Demographic dataset & Every Belgian citizen & $\begin{array}{l}\text { Nationality group, household } \\
\text { composition }\end{array}$ \\
\hline & $\begin{array}{l}\text { Socio-economic survey (SES) } \\
2001 \text { and Census } 2011\end{array}$ & $\begin{array}{l}\text { Every Belgian citizen, information } \\
\text { gathered from multiple external }\end{array}$ & $\begin{array}{l}\text { Highest attained education level, } \\
\text { occupation, housing comfort }\end{array}$ \\
\hline
\end{tabular}

security number (Census 2011)

Net income by category

Identified but not used in our research

Belgian Ministry of Health Minimal Hospital Dataset

Every hospital admission in non-

psychiatric general hospitals

Medical, nursery and personnel data for in-hospital care

cancer and TNM (tumour node metastasis) classification of malignant tumours [15].

However, the cancer registry data does not make it possible to distinguish between those who died 'from' cancer and those who died 'with' cancer. Additionally, since no similar registries were available to identify those who died with or from Alzheimer's disease and COPD we identified the death certificate data as a necessary additional database. Death certificate data in Belgium are collected by three administrations (corresponding to the three semi-autonomous regions in the country, i.e. Brussels, Flanders and Wallonia) and are integrated by Statistics Belgium into one national database for cause of death statistics. This database provides the causes of death and associated causes of death (coded in ICD-10 [10th revision of the International Statistical Classification of Diseases and Related Health Problems] codes) for all decedents.

Statistics Belgium also manages the national demographic database, derived from the population register [16] and containing for example the household composition of every citizen and data from the Socio-Economic Survey 2001 and Census 2011, nationwide full population surveys based on the tradition of population count [17]. The database contains information about the highest educational level attained, the last held occupation (as a measure of socio-economic position) and housing characteristics, which are all socio-economic factors that have been identified in previous studies as affecting end-of-life care patterns [18-20]. Finally a database containing fiscal data (i.e. net taxable household income), also managed by Statistics Belgium, was identified as providing additional socio-economic variables of influence on end-of-life care patterns.

For more specific clinical data, the Minimal Hospital dataset, providing clinical information associated with hospitalizations, was looked at for possible inclusion. This dataset has high quality data and provides diagnostic information (in ICD-codes), which allows for a more exact clinical description of the study population. It is however limited to in-hospital data, limiting the study population. Additionally, clinical information can be abstracted from health care claims data using specific algorithms. Obtaining cause of death information and using healthcare claims data makes up for the lack of clinical data. Therefore inclusion of the Minimal Hospital dataset was found to be unnecessary.

The combination of identified databases would provide information on formal health care and medication prescription, causes of death, main diagnosis (through the 
cancer diagnostic information of the cancer registry and algorithmic estimation methods in the IMA databases), and various relevant socio-demographic and socioeconomic information.

\section{Access procedures}

Two types of approval were needed for every database: (1) internal approval from database administrator organizations and (2) approval from the relevant Belgian Privacy Commission bodies.

(1)To obtain access to the IMA and cancer registry databases several steps are required. First, a declaration of interest needs to be set up between researchers and IMA and cancer registry programme managers. Research goals, databases, variables and linking possibilities (see Data linkage procedure) need to be discussed. After IMA and cancer registry programme managers agree on cooperation, the research project (research goals and requested data) is presented to IMA and cancer registry directory boards for approval. To obtain access to the databases administered by Statistics Belgium, no formal approval of the directory board is required, since Statistics Belgium is legally committed to providing data for research. Based on the requested data, variables and linking possibilities (see Data linkage procedure) the statisticians of Statistics Belgium deliver non-binding advice. Data requests should be filed directly to the Privacy Committee.

All involved partners then discuss the final selection of data and variables and initiate preparations for the linking procedure. The linking of the databases is a main issue for approval by the involved Privacy Commission bodies.

(2) We needed the approval of two separate national sectoral committees for privacy protection for access to the various databases and the database integrating all databases: the 'Sectoral Committee of Social Security and Health, Section Health' and the 'Statistical Supervisory Committee'. Both are subcommittees of the Belgian Commission for the Protection of Privacy. The former is responsible for privacy protection of health care data (IMA and cancer registry databases), the latter for privacy of national statistical data (Statistics Belgium databases). The application to the Sectoral Committee of Social Security and Health, Section Health consists of two phases. Phase 1 is the submission of the application and a first assessment by the committee in a plenary meeting. The primary investigator of the study presents the research goals and data linkage procedure to the committee at this plenary meeting. In our application special attention was given to the selection of different variables to receive data with sufficient detail for analysis, but at the same time reducing the risk of re-identification of deceased individuals and their families in order to preserve privacy. Changes in the linking procedure and storage on a separate server were requested (see section on data linkage for more details). Phase 2 is the formal approval during a second plenary meeting of the committee, after having received additional information from the applicant. In our case, the committee requested an additional risk analysis to ensure privacy of the included individuals, which was not requested in the first phase. Formal approval was granted only after a third plenary meeting. The full process took six months from application to formal approval.

The Statistical Supervisory Committee application procedure consists of one phase in which the application is assessed and discussed on a plenary meeting. Formal approval was granted after the first meeting.

\section{Data linkage procedure}

All eligible databases needed to be linked into one integrated database for analysis; a common unique identifier (i.e. social security number) made deterministic linking possible. Although the death certificate database does not contain this unique identifier, Statistics Belgium performed a linkage between the death certificate database and the national registry database based on date of birth, sex, and municipality of residence in order to include this unique identifier as a variable. Unique linkage was possible for $98.4 \%$ of deaths. ${ }^{1}$

For privacy reasons, Trusted Third Parties (TTPs) 'eHealth' and 'Crossroads Bank for Social Security (CBSS)' were responsible for the simple deterministic one-to-one record linkage of the IMA, cancer registry and Statistics Belgium databases. The linkage procedure (Fig. 1) consisted of 13 steps of data-coding or decoding and data transfers needed to ensure that none of the involved parties would have access to both the sensitive data and the social security numbers or to their own databases enriched with data from one of the other parties. Only the researchers have access to the complete linked database without unique identifiers using a Virtual Private Network (VPN) connection with secure token.

Linkage of all data for deaths in 2012 (including health care information about the two years prior to death) were completed in a first phase of the project. In a second phase all data for all deaths 2010-20xx are linked, where data from subsequent years will be added upon availability. A major consideration in the decision to adopt this phased approach is the size of the linked 


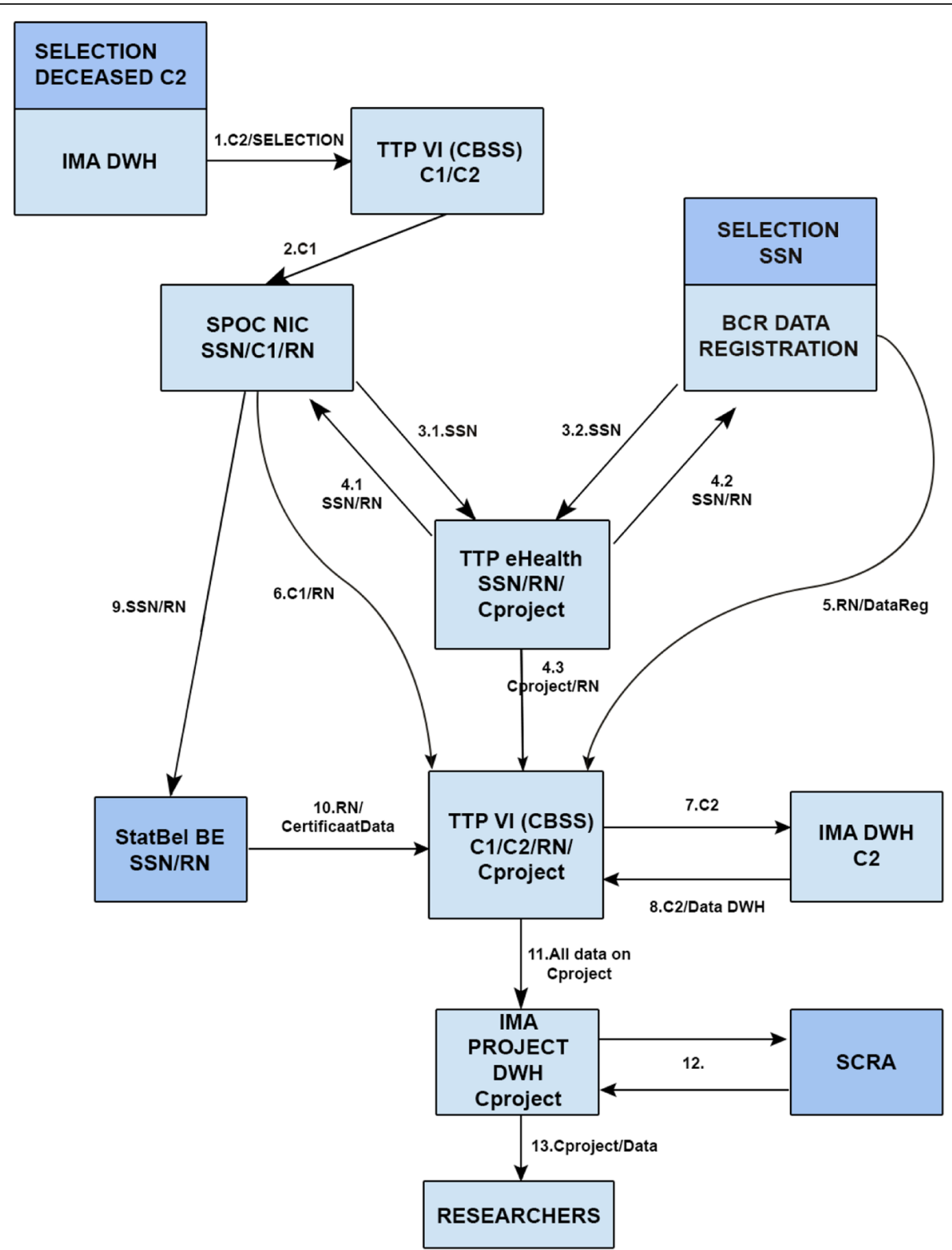

Fig. 1 (See legend on next page.) 
(See figure on previous page.)

Fig. 1 Step-by-step overview of linkage procedure. IMA DWH: InterMutualistic Agency Data Warehouse; TTP VI CBSS: Trusted Third Party Crossroads Bank for Social Security; SPOC NIC: Single Point of Contact National InterMutualistic College; BCR: Belgian Cancer Registry; TTP eHealth: Trusted Third Party eHealth; StatBel BE: Statistics Belgium; SCRA: Small Cells Risk Analysis; SSN: Social Security Number; C1/C2: coding 1/2; Explanatory note: The linkage procedure consisted of 13 steps (cf. arrows Fig. 1). Step 1: All cases from Belgian decedents since January 1, 2010 are selected in the IMA databases with their specific identifier coded (C2). These are then decoded (C1) by the TTP VI (CBSS). Step 2: The security officer of the National InterMutualistic College decodes the identifiers (C1) into actual social security numbers. Step 3.1: The IMA subset of social security numbers is sent by secure means to the separate TTP eHealth. Step 3.2: TTP eHealth receives the social security numbers from all cases in the Cancer Registry selected for the study (decedents since January 1, 2010). Step 4.1 and 4.2: To avoid any party from having access to both the sensitive data and the social security numbers, the established principle of random transport numbers (RN) is used. TTP eHealth assigned these RNs for the selected cases from IMA (4.1) and BCR (4.2) and provides these RNs to both data agencies in order to transmit the sensitive data safely to the TTP VI (CBSS). Step 4.3: TTP eHealth recodes the social security numbers into a final code that can be made available to the researchers (Cproject). These are sent, with the RNs as a cross reference coding, to the TTP VI (CBSS). Step 5: The selected cases and the corresponding requested data from the Cancer Registry are securely transmitted to the TTP VI (CBSS). Step 6: The selected IMA cases (but not yet the corresponding requested data) are securely transmitted to the TTP VI (CBSS). Step 7: The selected cases are transferred to the IMA datawarehouse (based on C2) so as to allow the selection of all data corresponding to these cases. Step 8: The selected cases and the corresponding requested data from IMA are securely transmitted to the TTP VI (CBSS). Step 9: The social security numbers and corresponding RNs are transferred safely to Statistics Belgium in order to allow selection of the correct cases. A social security number has already been attributed by Statistics Belgium to every case in the death certificate data (which do not contain the social security numbers) based on a deterministic linkage between the death certificate database and the national registry database based on date of birth, sex, and municipality of residence. Step 10: Statistics Belgium sends the requested data from the selected cases to TTP VI (CBSS) who links these with the data from IMA and Cancer Registry using the RNs. Step 11: The TTP VI (CBSS) recodes all data one final time based on the Cproject coding. Step 12: A small cells risk analysis is performed to minimize the risk of re-identification based on a combination of variables. Step 13: The complete linked database is stored on a separate IMA data server, which is only accessible to the researchers through a Virtual Private Network (VPN) connection with secure token

database. The linked database (deaths in one year) will be used for the initial analysis, after which a selection of variables and/or information can be made. Variables with too many missing data or variables that are inaccurate can be dropped. Additionally, the initial analysis will inform on what health care interventions or medications are suitable for further analyses. Finally, based on this first analysis phase, detailed information can be aggregated. The second and third delivery will therefore include more cases with more condensed information per case.

Since all databases depend on submission by individual organizations or institutions, a two-year delay is common. Linkage can only be initiated after all data are complete.

\section{Available information and data handling}

Variables selected in this study include data on health care use, prescribed medication, demographics, socio-economics and use of special reimbursement rules. A complete list of variables can be found in Additional file 1: Table S1.

Several steps were necessary to make the data analysis-ready:

1. In the IMA databases, health care and medication data are coded as nomenclature and Anatomical Therapeutic Chemical Classification (ATC) codes. In order to answer research questions, nomenclature numbers had to be interpreted and possibly aggregated by the researchers into meaningful categories.

2. Due to privacy concerns, no raw dates (e.g. date of birth, prescription date) were provided by database administrators. Dates of medication delivery or health service provision were therefore transformed into a number of days before death. Combinations of these recoded dates and nomenclature or ATC codes are used to determine whether certain interventions occur within a certain time period before death.

3. Since no data were provided on diagnosis in the current set of linked databases (only causes of death are available), algorithms were used to abstract diagnostic information from health care and medication prescription data. Algorithms were developed to identify people with COPD or Alzheimer's disease, based on treatments and medication received. The algorithms were developed using existing evidence [21-23] and were validated by medical experts and medical data experts from the IMA. They were then applied by the IMA, prior to the linking procedure, because data were used that were not available to the researchers; data provided to the researchers were limited to two years prior to death, while data used for identification of patients with Alzheimer's disease went back to six years before death. Combinations of the algorithmic identification of diagnosis and the causes of death (including the associated causes) can be used to identify relevant disease groups in the analyses.

\section{Discussion}

\section{Summary of main results}

In linking information from seven different datasets we managed to obtain a database that can provide information about patterns in the use, quality and costs of end of life care at the level of the full population and 
their associations with various clinical, sociodemographic, socio-economic and environmental factors. The process of obtaining this involved detailed identification of databases fitting the study aims, negotiation with and formal approval of three database administrators, three supervisory bodies and two national privacy commissions and eventual linking of all databases through two Trusted Third Parties (TTPs) using multiple encrypted social security numbers.

We believe that the described process can be particularly helpful to researchers in other countries in compiling similar population-level databases on end-of-life care. A number of considerations (limitations, strengths and opportunities) and recommendations can be made based on our experience.

\section{Limitations of our study}

Our study involved a systematic and thorough exploration of how several databases providing information on end-of-life care can be accessed, handled and linked into an integrated and enriched database. However, an important limitation is that linkage with information on patient-related outcomes of healthcare services, such as specific Patient Reported Outcome Measures (PROMs) was not explored in our study. Even though PROMs are important indicators to evaluate whether increased healthcare expenditure results in better health outcomes, their inclusion in a population-level database is only meaningful if there is sufficient standardization in the measurement methodology. In Belgium, a common coding system for PROMs is lacking and would be timeconsuming to perform. [24] Future efforts could be made to include PROMs at a population level.

\section{Opportunities of the collected database}

Our efforts resulted in a population-level database with detailed information about formal end-of-life care, the costs of care and demographic, socio-economic and diagnostic information on decedents. The opportunities provided by such a database to study use, quality and costs of end-of-life care are considerable. The main overall advantage is that data are population-level and therefore not subject to sample bias such as in surveys or medical records studies of selected groups of patients. Compared to primary data collection, using linked routinely collected databases as in our case is less expensive and less time-consuming. In the end-of-life care context specifically, primary data collection can be burdensome for patients and caregivers. Furthermore, in routinely collected databases, high-quality data are available on the spot, although they are not deliberately collected for research aims.

Although the linked database does not include certain types of information that are important in evaluating quality of care, such as patient-specific preferences of care, psycho-social information, patient or family reported outcomes and experiences or information about pain and symptom management or communication aspects [25], the full-population data have the potential to provide robust and population-level measures of the quality of end-of-life care using specific claims-based quality indicators. These quality indicators, e.g. mapping inappropriate end-of-life care, have been used in various studies as measures for the quality of end-of-life care [26]. If preceded by an adequate validation process, they can provide a detailed image of the quality of end-of-life care by regions or health care providers [26]. The linked database also contains data on all direct medical costs and reimbursed service and medication use, which offers opportunities to study direct medical costs and patterns in the use of specific end-of-life care for full populations. Policy measures that support palliative care include financial compensation directed towards the patient (e.g. monthly lump sum to cover additional costs for palliative home patients). Using this database, patterns in the uptake of these measures can be mapped and compared between population or pathology groups. As the linked database contains individual data, these data can be aggregated on multiple levels, which makes longitudinal, disease-, treatment- or provider-specific analyses possible. As a result, it is also possible to evaluate the influence of certain policy measures and governmental support programmes. Without the rich population-level data we collected it would be impossible to answer these example research questions without facing major issues of reliability, generalizability, feasibility and costs.

Data allow us to follow back the treatment history and costs of those treatments up to two years before death. Although a shorter period before death may be sufficient to study several aspects of end-of-life care in specific disease groups, for other (particularly noncancer) longer time periods are warranted. The decision to request all health care and medication data up to two years before death (irrespective of when the diagnosis was made) was also made for practical reasons as going further back would substantially increase storage and analysis requirements.

\section{Limitations of the collected database}

A limitation of these types of routinely collected population-level data is that services not covered by insurers are not included. Researchers from other countries that wish to compile a similar database need to remember that what is not covered by insurance (and hence not found in the data) may be country- or even region-specific. In Belgium, data are relatively complete, for health care services in the hospital, nursing homes and at home. Nevertheless use of certain services cannot be 
identified because there is no individual reimbursement (e.g. mobile hospital palliative care teams) or such reimbursement is not regulated or generalized (e.g. consultations of a psychologist). Secondly, total out-of-pocket spending is not available in the integrated database. This results in an overall underestimation of the total cost of end-of-life care. Nevertheless, administrative data are an essential source of information for studies on the financial burden of end-of-life care for the health care budget and are valuable for policymakers in informing their decisions on health care policy [8].

\section{Considerations and actions for researchers considering similar database constructions (Table 2)}

The linkage process is crucial in obtaining a useful population-level database. It enables the enriching of the population-level data on formal end-of-life care and the costs of that care with putative demographic, socioeconomic and diagnostic information for the study of end-of-life care patterns. This allows the development of explanatory models and the provision of public health information to policy makers, for example on social differences and differences between pathology groups. It can support discussions on the organization of the health care system, based for instance on possible existing inequities.

The flip side is that the linking can create additional difficulties in the process of obtaining the data. While deterministic linking is relatively easy to complete on a technical level (even without identical unique identifiers a deterministic linking is possible based on a combination of variables), the main challenges for researchers lie in the fact that 1) several separate organizations have to be convinced to cooperate and 2) special attention needs to be given to privacy-related issues.

Databases across health and social care may not always contain a unique identifier variable, or not always contain accurate and fully available information that allows identifying unique persons. In such cases where the possibility to perform deterministic linkage is limited the method of probabilistic linking can present a solution. In this approach the likelihood of a correct linking is calculated and a linking is done when the likelihood is sufficiently large [27]. Several tools have been developed to perform this probabilistic linking [28]. Nevertheless, a lack of accurate and fully available personal identifiable information constrains a probabilistic linking method.

A final consideration for researchers who wish to have access to similar data in their country is that establishing and maintaining good relationships with database administrators is crucial. Gaining access to administrative data is an iterative process that requires a lot of preparatory work. Database administrators are the researchers' access points to the data and have all the information about internal procedures. Strict procedures need to be

Table 2 Considerations for researchers planning to link databases

\begin{tabular}{|c|c|}
\hline Topics & Considerations \\
\hline \multirow[t]{6}{*}{ Exploring relevant databases } & Are my research questions clear and well-defined? What data are needed to answer them? \\
\hline & What is/are my study population(s)? What data are needed to identify it? \\
\hline & What database(s) contains the core data and could thus be selected as a starting point? \\
\hline & $\begin{array}{l}\text { When a starting database is chosen, what data are lacking to fully address the research questions? } \\
\text { Where can we find them? }\end{array}$ \\
\hline & $\begin{array}{l}\text { How can we establish contact with the database administrators of the databases? Obtain principal } \\
\text { approval from all administrators (e.g. by presenting the study to the board of directors) }\end{array}$ \\
\hline & What is the cost associated with each database? \\
\hline \multirow[t]{5}{*}{ Variable selection } & What specific variables do we need from the selected databases to answer our research questions? \\
\hline & Are the variables we want available and linkable between the different databases? \\
\hline & $\begin{array}{l}\text { Does the preferred selection of variables complicate the linking procedure considerably? Balance the } \\
\text { gain in information with the increase in complexity and time. }\end{array}$ \\
\hline & $\begin{array}{l}\text { What is the required level of detail for each variable? Balance the preferred level with what is allowed in } \\
\text { terms of data protection (e.g. through small cells risk analysis to determine risk of re-identification based } \\
\text { on a combination of variables) }\end{array}$ \\
\hline & Do we have sufficient storage capacity and analysis hardware to store and analyze all the data we want? \\
\hline \multirow[t]{2}{*}{ Access procedures } & What ethical and privacy procedures need to be followed to link and access the selected database? \\
\hline & What technical procedures need to be followed to link and access the selected databases? \\
\hline \multirow[t]{3}{*}{ Infrastructure } & $\begin{array}{l}\text { How will data be stored safely? Is infrastructure provided by researchers or by database administrators? } \\
\text { What is the cost for this infrastructure? }\end{array}$ \\
\hline & How will data be protected? Physical and digital protection need to be guaranteed. \\
\hline & $\begin{array}{l}\text { How can data be accessed in a safe and easy way? What hardware and software do we need to access } \\
\text { and analyze the requested data? }\end{array}$ \\
\hline
\end{tabular}


followed, in close cooperation with database administrators. We were able to arrange an updated dataset where data from subsequent years will be added upon availability in the same approval and agreement, which limits the time of going through all necessary permissions each time an updated dataset is needed. Since administrative data are often not gathered with the intention of research, or only for internal use, the process of making the data analysis-ready can take time. Researchers must adapt to how data are registered and stored, before they can effectively use them for research.

\section{Conclusion}

Linking and accessing various routinely collected population-level databases involves challenges but offers substantial opportunities to study patterns in the use, quality and costs of end-of-life care both in the full population and for specific diagnostic groups. This study has identified that it is possible to combine data from different databases in order to obtain a rich database for such analysis, including information about all reimbursed care and medication as well as disease, demographic, socio-economic and environmental information. While some aspects may be specific to the Belgian context, our study has a much broader application as most developed countries collect similar population-level databases. The process described in our study can be a helpful aid for researchers in these countries to compile similar data and eventually develop an international comparative end-of-life care research agenda using administrative health care data.

\section{Endnote}

${ }^{1}$ Between 1998 and 2013, 1,662,550 of 1,689,740 deaths were matched (98.39\%).

\section{Additional file}

Additional file 1: Table S1. Complete list of variables in the linked dataset (IMA - Statistics Belgium - BCR). (DOCX $22 \mathrm{~kb}$ )

\section{Abbreviations}

ATC: Anatomical therapeutic chemical classification; CBSS: Crossroads bank for social security; COPD: Chronic obstructive pulmonary disease; ICD10: International statistical classification of diseases and related health problems; IMA: Inter mutualistic agency; PROMS: Patient reported outcome measures; TNM: Tumour node metastasis; TTP: Trusted third party; VPN: Virtual private network

\section{Acknowledgements}

The following persons contributed to the preparations for this project: Dirk De Kesel (InterMutualistic Agency), Karen Vos (Belgian Cancer Registry), Youri Baeyens (FPS Economy).

We thank Jane Ruthven for manuscript editing.

\section{Funding}

This study is part of a research study funded by the Research Foundation Flanders (FWO grant number G012414N) and the Government Agency for Innovation by Science and Technology (IWT-SBO grant number 140009).

\section{Availability of data and materials}

No dataset was used in this research article. All database administrators can be contacted freely.

\section{Authors' contributions}

AM, RDS and KF have equally contributed as first authors. All authors have contributed to the design of the study. All authors have been involved in critically revising the first draft and read and approved the final manuscript.

\section{Competing interests}

The authors declare that they have no competing interests.

Consent for publication

Not applicable.

\section{Ethics approval and consent to participate}

In accordance with Belgian law, approvals for access to the various databases and the database integrating all databases were obtained from two separate national sectoral committees for privacy protection: the 'Sectoral Committee of Social Security and Health, Section Health' and the 'Statistical Supervisory Committee'. Both are subcommittees of the Belgian Commission for the Protection of Privacy. Additionally, the ethics committee of the Ghent University Hospital provided approval (B670201422382).

\section{Author details}

${ }^{1}$ End of Life Care Research Group, Vrije Universiteit Brussel (VUB), Brussels, Belgium \& Ghent University, Ghent, Belgium. ${ }^{2}$ Interuniversity Centre for Health Economics Research (I-CHER), Ghent University, Ghent, Belgium. ${ }^{3}$ Department of medical oncology, Ghent University Hospital, Ghent, Belgium. ${ }^{4}$ InterMutualistic Agency, Brussels, Belgium. ${ }^{5}$ Belgian Cancer Registry, Brussels, Belgium. ${ }^{6}$ Statistics Belgium, Brussels, Belgium.

Received: 17 May 2016 Accepted: 11 October 2016

Published online: 18 October 2016

\section{References}

1. Cohen, J. \& Deliens, L. A Public Health Perspective on End of Life Care. Oxford: Oxford University Press, 2012.

2. Riley GF. Administrative and claims records as sources of health care cost data. Med Care. 2009:47:S51-55.

3. Benchimol El, et al. Development and use of reporting guidelines for assessing the quality of validation studies of health administrative data. J Clin Epidemiol. 2011;64:821-9.

4. Wilchesky M, Tamblyn RM, Huang A. Validation of diagnostic codes within medical services claims. J Clin Epidemiol. 2004;57:131-41.

5. Brownell MD, Jutte DP. Administrative data linkage as a tool for child maltreatment research. Child Abuse Negl. 2013;37:120-4.

6. Billings, J. Using Administrative Data To Monitor Access, Identify Disparities, and Assess Performance of the Safety Net. Agency for health care research and quality (2003). Available at: http://archive.ahrq.gov/data/safetynet/ billings.htm. (Accessed: 21st January 2016)

7. Blais C, et al. Quebec Integrated Chronic Disease Surveillance System (QICDSS), an innovative approach. Chronic Dis Inj Can. 2014:34:226-35.

8. Murray SA, Kendall M, Boyd K, Sheikh A. Illness trajectories and palliative care. BMJ. 2005:330:1007-11.

9. Jensen $\mathrm{PB}$, Jensen $\sqcup$, Brunak S. Mining electronic health records: towards better research applications and clinical care. Nat Rev Genet. 2012;13:395-405.

10. Murdoch TB, Detsky AS. The inevitable application of big data to health care. JAMA. 2013;309:1351-2.

11. Allan DE, Stajduhar Kl, Reid RC. The uses of provincial administrative health databases for research on palliative care: Insights from British Columbia, Canada. BMC Palliat Care. 2005:4:2.

12. Waien SA. Linking large administrative databases: a method for conducting emergency medical services cohort studies using existing data. Acad Emerg Med Off J Soc Acad Emerg Med. 1997:4:1087-95. 
13. Hunt R, McCaul K. A population-based study of the coverage of cancer patients by hospice services. Palliat Med. 1996;10:5-12.

14. Johnston $\mathrm{GM}$, et al. Identifying potential need for cancer palliation in Nova Scotia. Can Med Assoc J. 1998;158:1691-8.

15. Belgian Cancer Registry. Manuel Web Based Cancer Registration WBCR. (2013).

16. FOD Binnenlandse Zaken - Algemene directie Instellingen en Bevolking Rijksregister - Rijksregister - IBZ Instellingen en Bevolking. Rijksregister (2014). Available at: http://www.ibz.rrn.fgov.be/nl/rijksregister/. (Accessed: 6th April 2016)

17. Statistics Belgium. Census 2011 - Statistics \& Analyses. Census 2011 (2013). Available at: http://statbel.fgov.be/en/statistics/surveys-methodology/ census/Census2011/. (Accessed: 6th April 2016)

18. Hanchate A, Kronman AC, Young-Xu Y, Ash AS, Emanuel E. Racial and Ethnic Differences in End-of-Life Costs: Why Do Minorities Cost More Than Whites? Arch Intern Med. 2009;169:493.

19. Huang C-Y, Hung Y-T, Chang C-M, Juang S-Y, Lee C-C. The association between individual income and aggressive end-of-life treatment in older cancer decedents in Taiwan. PloS One. 2015;10:e0116913.

20. Chang C-M, et al. Low socioeconomic status is associated with more aggressive end-of-life care for working-age terminal cancer patients. Oncologist. 2014;19:1241-8.

21. Waldemar $\mathrm{G}$, et al. Recommendations for the diagnosis and management of Alzheimer's disease and other disorders associated with dementia: EFNS guideline. Eur J Neurol. 2007;14:e1-26.

22. Schmitt FA, van Dyck CH, Wichems CH, Olin JT. Memantine MEM-MD-02 Study Group. Cognitive response to memantine in moderate to severe Alzheimer disease patients already receiving donepezil: an exploratory reanalysis. Alzheimer Dis Assoc Disord. 2006;20:255-62.

23. Smidth M, Sokolowski I, Kærsvang L, Vedsted P. Developing an algorithm to identify people with Chronic Obstructive Pulmonary Disease (COPD) using administrative data. BMC Med Inform Decis Mak. 2012;12:38.

24. Black, N. et al. Patient-reported outcomes: pathways to better health, better services, and better societies. Qual. Life Res. Int. J. Qual. Life Asp. Treat. Care Rehabil. (2015). doi:10.1007/s11136-015-1168-3

25. Grunfeld E, et al. Towards using administrative databases to measure population-based indicators of quality of end-of-life care: testing the methodology. Palliat Med. 2006;20:769-77.

26. Earle CC, et al. Identifying potential indicators of the quality of end-of-life cancer care from administrative data. J Clin Oncol Off J Am Soc Clin Oncol. 2003;21:1133-8

27. Newcombe, H. B. Handbook of record linkage: methods for health and statistical studies, administration, and business. (Oxford University Press, Inc., 1988)

28. Aldridge RW, Shaji K, Hayward AC, Abubakar I. Accuracy of Probabilistic Linkage Using the Enhanced Matching System for Public Health and Epidemiological Studies. PloS One. 2015;10:e0136179. doi:10.1371/journal. pone.0136179.

\section{Submit your next manuscript to BioMed Central and we will help you at every step:}

- We accept pre-submission inquiries

- Our selector tool helps you to find the most relevant journal

- We provide round the clock customer support

- Convenient online submission

- Thorough peer review

- Inclusion in PubMed and all major indexing services

- Maximum visibility for your research

Submit your manuscript at www.biomedcentral.com/submit 